of underweight $(7 / 9(78 \%))$, healthy $(28 / 46 \quad(61 \%))$, overweight $(14 / 23(61 \%))$ and obese $(6 / 12 \quad(50 \%) \quad p=0.642)$. Patients with $>5 \%$ weight loss $(13 / 101(13 \%))$ or suboptimal HGS (56/101 (55\%)) were significantly more likely to be at nutrition risk using IBD-NST, MUST and SGA. Patients with suboptimal MAMC (10/66 (15\%)) were not significantly more likely to be at nutrition risk using IBD-NST or MUST.

Conclusion The IBD-NST identifies more patients with high nutrition risk and places less importance on BMI than SGA or MUST. Finally, we confirm that BMI is a poor indicator of HGS in IBD supporting a reduced importance in identifying nutrition risk. Further work is underway to test the repeatreliability the IBD-NST.

\section{PTH-136 INFLAMMTORY BOWEL DISEASE PATIENTS EXPRESS HIGHER LEVELS OF CIRCULATING GALECTINS-1 AND -3 THAN HEALTHY PEOPLE}

${ }^{1}$ Sreedhar Subramanian, ${ }^{2}$ Tony Yu*, ${ }^{3}$ Susanna Dodd, ${ }^{4}$ Lugang Yu. ${ }^{1}$ Royal Liverpool University Hospital, Liverpool, UK; ${ }^{2}$ School of Medicine, University of Edinburgh, Edinburgh, UK; ${ }^{3}$ Department of Biostatistics, Institute of Translational Medicine, University of Liverpool, Liverpool, L69 3GS, UK; ${ }^{4}$ Institute of Translational Medicine, University of Liverpool, Crown Street, Liverpool L69 3BX, UK

\subsection{6/gutjnl-2019-BSGAbstracts.195}

Introduction Non-invasive biomarkers currently available in clinic in (i) accurately diagnosing IBD and (ii) in determining disease activity in established IBD all show poor sensitivity and substantial limitation. In this study, we investigated whether levels of serum galectins, a family of 15 galactosidebinding proteins, could be used as potential non-invasive biomarkers for IBD diagnosis and for determining the disease activity.

Methods A single centre prospective cross-sectional study was conducted in 168 IBD patients (97 CD and $71 \mathrm{UC}$ ) and 40 healthy controls. Disease activity was assessed using HarveyBradshaw Index (HBI) for CD and simple clinical colitis activity index (SCCAI) for UC. Active disease was defined as $\mathrm{HBI}>5$ and SCCAI $>2$. Serum levels of galectins- $1,-2,-3,-4$, -7 and -8 was analysed by ELISA and compared with that in active and non-active IBD patients and healthy controls.

Results Serum levels of galectins- 1 and -3 , but not galectins-2, $-4,-7$ and -8 , were significantly higher in patients with CD $(\mathrm{p}=0.0001$ and $\mathrm{p}=0.0003$ for galectin- 1 and -3 , respectively) and UC $(p=0.0002$ and $p=0.0001$ for galectin- 1 and -3 , respectively) as compared to healthy controls. Twenty seven $(27.8 \%)$ CD patients and 41 UC patients (57.8\%) had clinically active disease. None of the serum galectins including galectins -1 and -3 showed significantly different in patients with active UC or CD compared to patients in clinical remission. Serum CRP was significantly higher in patients with active UC (mean $\pm \mathrm{SD}, 7.3 \pm 12.5 \mathrm{mg} / \mathrm{L}$ ) compared to those in remission (4.8 $\pm 14.5 \mathrm{mg} / \mathrm{L}, \mathrm{P}=0.047)$. Similarly, serum CRP was higher in patients with active CD $(4.6 \pm 8.2 \mathrm{mg} / \mathrm{L})$ compared to those in remission $(10.2 \pm 12.1 \mathrm{mg} / \mathrm{L}, \mathrm{P}=0.047)$.
Conclusions Serum levels of galectins-1 and -3 were significantly higher in IBD patients compared to healthy people and this difference however could not separate active from inactive IBD.

\section{PTH-137 PHOSPHARE STUDIES: IMPORTANT CHANGES IN PHOSPHATE HOMEOSTASIS AND BONE METABOLISM AFTER IV IRON}

${ }^{1}$ Tariq Iqbal ${ }^{*}$, Myles Wolf, Philip Schaffalitzky de Muckadell, Heinz Zoller. 'Queen Elizabeth Hospital Birmingham, Birmingham, UK; ${ }^{2}$ Duke University School of Medicine, North Carolina, USA; ${ }^{3}$ Pharmacosmos A/S, Holbaek, Denmark; ${ }^{4}$ Medical University of Innsbruck, Innsbruck, Austria

\subsection{6/gutjnl-2019-BSGAbstracts. 196}

Introduction Iron deficiency anaemia (IDA) is the most common extraintestinal complication of inflammatory bowel disease (IBD), and IV iron is commonly used to treat IDA in IBD. Of the two commonly used IV iron preparations, ferric carboxymaltose (FCM) and iron isomaltoside IIM, there is evidence that FCM can cause hypophosphatemia due to FGF23 mediated renal phosphate wasting, which has been associated with osteomalacia; the comparative effects of IIM are unknown.

Methods In two identical, open-label trials, 245 adults with IDA randomly received $(1: 1)$ IIM $(1 \times 1000 \mathrm{mg})$ or FCM $(2 \times 750 \mathrm{mg} ; 1$ week apart). We compared the incidence, severity and duration of hypophosphatemia, and effects on fractional phosphate urinary excretion (FEPO4), FGF23, parathyroid hormone $(\mathrm{PTH})$, vitamin $\mathrm{D}$ (VitD), and bone turnover biomarkers in blood and urine.

Results In a pooled analysis, the incidence of hypophosphatemia $<2 \mathrm{mg} / \mathrm{dL}$ was markedly higher in the FCM vs. IIM group $(74.4 \%$ vs. $8.0 \%, \mathrm{p}<0.0001)$. Hypophosphatemia persisted at day 35 in $43.0 \%$ of FCM-treated patients compared to $0.9 \%$ of IIM-treated patients $(\mathrm{p}<0.0001)$. Severe hypophosphatemia $\leq 1 \mathrm{mg} / \mathrm{dL}$ occurred in $11.3 \%$ of FCM patients compared to $0.0 \%$ of IIM-treated patients $(\mathrm{p}<0.0001)$. FCM infusion significantly increased intact FGF23 compared to IIM $(\mathrm{p}<0.0001)$ : FGF23 after FCM rose from $49.9 \mathrm{pg} / \mathrm{mL}$ at baseline to $149.5 \mathrm{pg} / \mathrm{mL}$ one day post-infusion; and to $327.9 \mathrm{pg} / \mathrm{ml}$ by day 8.The corresponding figures for IIM were $59.9 \mathrm{pg} / \mathrm{mL}$ at baseline, $58.3 \mathrm{pg} / \mathrm{mL}$ by day 1 and 66.9 $\mathrm{pg} / \mathrm{mL}$ by day 8 . Compared to treatment with IIM, FCM significantly: increased FEPO4; decreased serum 1,25-(OH)2 vitD; decreased ionized calcium; and increased PTH, which persisted through day 35. Post-FCM changes were accompanied by significant increases in total and bone specific alkaline phosphatase that persisted through day 35. Correction of IDA was comparable between the two treatments. Significantly fewer adverse drug reactions occurred with IIM than with FCM. Serious or severe hypersensitivity reactions occurred in $0.8 \%$ in the IIM group and $1.7 \%$ in the FCM group.

Conclusion Compared to IIM, FCM-induced high rates of FGF23-mediated hypophosphatemia, which was frequently 
severe and often persisted for $>35$ days. FCM, but not IIM, induced changes in vitD and calcium homeostasis that triggered secondary hyperparathyroidism, which likely contributed to persistence of hypophosphatemia. Alteration of bone metabolism after FCM administration may be of great clinical significance particularly for IBD patients who often have low vit D and regularly receive iv iron.

\section{PTH-138 COLORECTAL CANCER, COLECTOMY AND INFLAMMATORY BOWEL DISEASE ACTIVITY FOLLOWING LIVER TRANSPLANTATION IN PRIMARY SCLEROSING CHOLANGITIS}

${ }^{1}$ Tom Thomas*, ${ }^{2}$ Rachel Cooney, 2,3 Tariq Iqbal, 2,35ubrata Ghosh, 2,3 James Ferguson, ${ }^{4}$ ProfGideon Hirschfield, $2,3,5$ Palak Trivedi. ${ }^{1}$ University Of Oxford, Oxford, UK; ${ }^{2}$ University Hospitals Birmingham, Birmingham, UK; ${ }^{3}$ National Institute for Health Research (NIHR) Birmingham Biomedical Research Centre (BRC), Birmingham, UK; ${ }^{4}$ Toronto Centre for Liver Disease, University of Toronto, Toronto, Canada; ${ }^{5}$ University of Birmingham, Birmingham, UK

\subsection{6/gutjnl-2019-BSGAbstracts.197}

Introduction Primary sclerosing cholangitis[PSC] is the classical hepatobiliary manifestation of inflammatory bowel disease[IBD] for which liver transplantation[LT] is the only curative therapy.In this study,we provide pooled incidence rates[IR] and time trends of (1)colorectal cancer[CRC], (2)flares in IBD activity and (3)colectomy rates post LT, through systematic review and meta-analysis.

Methods A systematic literature search of Medline and Embase was undertaken to identify pertinent studies from 1981 to 2014.Included studies were assigned to one or more of the three analytical streams. The 'meta' package (R Studio (V.1.1.463)) and Revman was used to pool IRs and HRs from individual studies using a random effects model respectively.

Results 42 studies qualified for inclusion in the systematic review.20 studies detailing the clinical course of 1994 patients (cumulative 9874 patient years(PY)) were pooled to assess the occurrence of dysplasia or CRC(combined endpoint) and CRC only; yielding an IR of 14.97 (95\%CI9.74-23.02) and 9.21 (95\%CI6.01-14.09) per 1000 PY,respectively. Heterogeneity was considerable $\left(\mathrm{I}^{2}=86 \%\right)$. The incidence of post LT CRC was seen to be decreasing over the past decade(figure 1A).The estimated colectomy rate following OLT was IR 23.18(95\% CI16.74-32.08, I ${ }^{2}=84 \%$ ) per 1000 PY. IR for colectomy due to dysplasia/CRC was 11.25 per 1000 PY(95\%CI6.43-19.68) and decreasing over time(figure 1B).By contrast, deterioration in IBD activity necessitating colectomy was noted for IR 13.26 per 1000 PY(95\% CI 9.95-17.66), with no change in the incidence rate over study duration(figure 1C).9 studies reported on post LT clinical course of IBD according to endoscopy findings or need for escalation in IBD therapy.27.6\% of patients $(n=584)$ experienced deterioration in IBD activity after LT.The effect of ursodeoxycholic acid [UDCA] on the risk of CRC after LT was examined by 3 studies.Due to data availability,only 2 studies could be pooled. Use of UDCA increased the risk of CRC post LT in the PSC cohort; HR 2.90 (95\%CI 1.37-6.11).Studies examining the impact of UDCA on colectomy and IBD activity post-LT were inconclusive.

Conclusion The heightened risk of CRC mandates ongoing colonoscopic surveillance in the PSC/IBD LT population, although the incidence appears to be decreasing.Identification of risk predictors impacting the course of IBD is of critical importance,given that deterioration in activity appears to be the principal indication for colectomy post LT.

\section{Liver}

\section{Orals}

\section{OTH-10 THERAPEUTIC INTERLEUKIN 4 MODULATES MONOCYTE DYNAMICS AND ACCELERATES REPAIR FOLLOWING ACUTE LIVER INJURY}

${ }^{1}$ Ruairi Lynch*, ${ }^{1}$ Calum Bain, ${ }^{1}$ Cathy Hawley, ${ }^{2}$ Professor Stuart Forbes, 'Steve Jenkins. 'Queen's Medical Research Institute, University of Edinburgh, Edinburgh, UK; ${ }^{2}$ Centre for Regenerative Medicine, University of Edinburgh, Edinburgh, UK

\subsection{6/gutjnl-2019-BSGAbstracts.198}

Introduction Acute liver failure has significant mortality, and in its most severe form, the only treatment is liver
A) Colorectal cancer and dysplasia

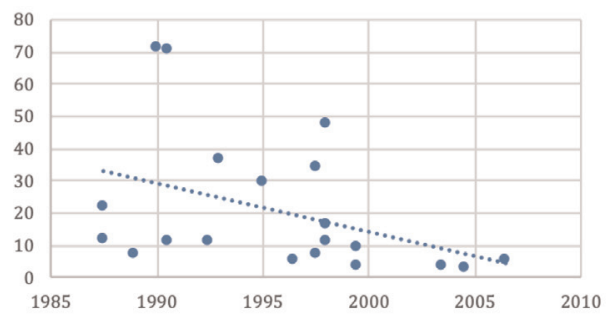

C) Colectomy due to IBD activity refractory to medical therapy

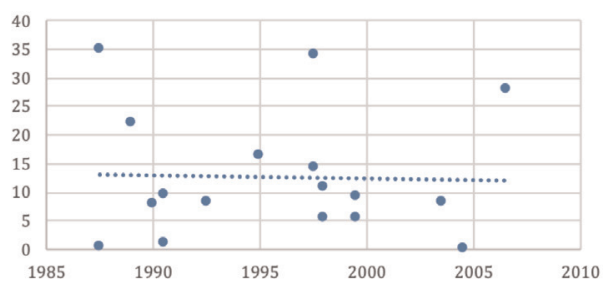

B) Colectomy due to CRC

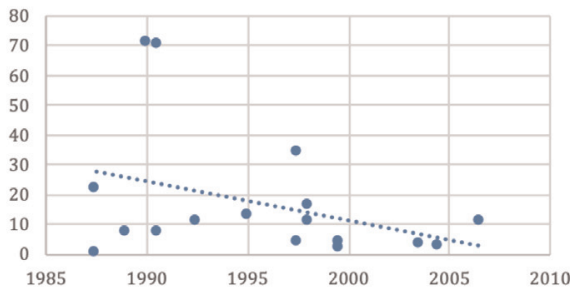

Abstract PTH138 Figure 1 Time trend analysis of incidence rates (per 1,000 pt.-yrs.) of IBD complications in PSC liver transplantation 